\title{
Versatile Functionalization of Carbon Nanomaterials by Ferrate(VI)
}

Cite as

Nano-Micro Lett.

(2020) 12:32

Received: 11 October 2019

Accepted: 1 December 2019

Published online: 21 January 2020

(C) The Author(s) 2020

\author{
Ying Zhou ${ }^{1}$, Zhao-Yang Zhang ${ }^{1}{ }^{凶}$, Xianhui Huang ${ }^{1}$, Jiantong $\mathrm{Li}^{2}$, Tao $\mathrm{Li}^{1}{ }^{凶}$ \\ $\triangle$ Zhao-Yang Zhang, zy_zhang@sjtu.edu.cn; Tao Li, litao1983@sjtu.edu.cn \\ 1 School of Chemistry and Chemical Engineering, Key Laboratory of Thin Film and Microfabrication \\ (Ministry of Education), Shanghai Jiao Tong University, Shanghai 200240, People's Republic of China \\ 2 School of Electrical Engineering and Computer Science, KTH Royal Institute of Technology, Electrum 229, \\ 16440 Kista, Sweden
}

\section{HIGHLIGHTS}

- Various forms of carbon nanomaterials are selected as substrates to clear the mist in understanding the reactivity/utility of ferrate(VI) in oxidizing carbon nanomaterials.

- It unravels a modest reactivity of ferrate(VI) in liquid phase that only oxidizes the active defects on carbon surface and a powerful oxidizing ability in solid state that can open the inert $\mathrm{C}=\mathrm{C}$ bonds in carbon lattice.

- Respective benefit and limitation of the wet and dry approaches using ferrate(VI) in functionalizing carbon nanomaterials are discussed.

ABSTRACT As a high-valent iron compound with $\mathrm{Fe}$ in the highest accessible oxidation state, ferrate(VI) brings unique opportunities for a number of areas where chemical oxidation is essential. Recently, it is emerging as a novel oxidizing agent for materials chemistry, especially for the oxidation of carbon materials. However, the reported reactivity in liquid phase $\left(\mathrm{H}_{2} \mathrm{SO}_{4}\right.$ medium $)$ is confusing, which ranges from aggressive to moderate, and even incompetent. Meanwhile, the solid-state reactivity underlying the "dry" chemistry of ferrate(VI) remains poorly understood. Herein, we scrutinize the reactivity of $\mathrm{K}_{2} \mathrm{FeO}_{4}$ using fullerene $\mathrm{C}_{60}$ and various nanocarbons as substrates. The results unravel a modest reactivity in liquid phase that only oxidizes the active defects on carbon surface and a powerful oxidizing ability in solid state that can

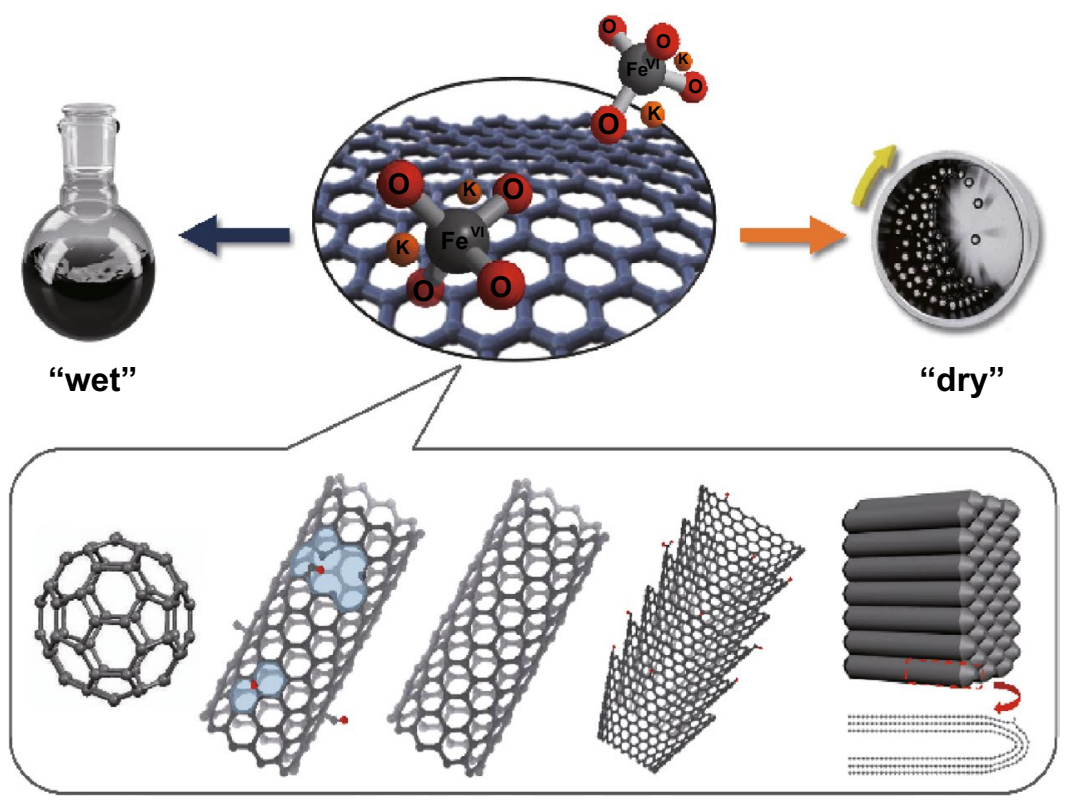
open the inert $\mathrm{C}=\mathrm{C}$ bonds in carbon lattice. We also discuss respective benefit and limitation of the wet and dry approaches. Our work provides a rational understanding on the oxidizing ability of ferrate(VI) and can guide its application in functionalization/transformation of carbons and also other kinds of materials.

KEYWORDS Ferrate(VI); Reactivity; Carbon nanomaterials; Oxidation 


\section{Introduction}

Nature utilizes $\mathrm{Fe}^{\mathrm{IV}}=\mathrm{O}$ and $\mathrm{Fe}^{\mathrm{V}}=\mathrm{O}$ complexes as the active centers for a number of important enzymatic oxidations, which motivates many fundamental studies on the properties of high-valent iron compounds, especially their chemical and biological reactivities [1-8]. $\mathrm{Fe}^{\mathrm{VI}}$, the highest accessible oxidation level of iron, generally exists in the form of ferrate(VI) ion $\left(\mathrm{FeO}_{4}{ }^{2-}\right)$ with four $\mathrm{Fe}^{\mathrm{VI}}=\mathrm{O}$ bonds. Ferrate(VI) possesses powerful oxidizing ability, as revealed by its higher redox potentials (up to $+2.2 \mathrm{~V}$ in acidic conditions) than those of most traditional oxidants [9-12]. Together with the environmentally benign nature, ferrate(VI) compounds (commonly $\mathrm{K}_{2} \mathrm{FeO}_{4}$ ) have been considered as promising oxidizing agents in several areas, including water remediation [13-17], organic synthesis [18-21], high-capacity battery [22-24] and $\mathrm{O}_{2}$ evolution [25-29].

Recent years have witnessed an emerging role of ferrate(VI) in materials science [30-34], where ferrate(VI)-enabled oxidative functionalization/transformation of a target material is a key step toward functional applications. Of particular interest is the oxidation treatment of carbon materials [35-41]. Peng et al. [35] reported the first example of applying $\mathrm{K}_{2} \mathrm{FeO}_{4}$ for the oxidation/ exfoliation of graphite in concentrated $\mathrm{H}_{2} \mathrm{SO}_{4}$. Highly water-soluble single-layer graphene oxide was produced in $1 \mathrm{~h}$ at room temperature, which indicated the extraordinary oxidizing ability of $\mathrm{K}_{2} \mathrm{FeO}_{4}$ in $\mathrm{H}_{2} \mathrm{SO}_{4}$ medium. But soon after, Sofer and coworkers [36] provided completely opposite results, showing such liquid-phase oxidation was impossible for graphite, attributed to the extreme instability of $\mathrm{K}_{2} \mathrm{FeO}_{4}$ in acidic environments. Nevertheless, some other studies suggested the moderate oxidation effects of $\mathrm{K}_{2} \mathrm{FeO}_{4}$ by the production of graphite/graphene oxides with relatively low degrees of oxidation $[37,38]$ and by the result of nondestructive oxidation of carbon nanotubes (CNTs) [39]. Therefore, the reactivity of $\mathrm{K}_{2} \mathrm{FeO}_{4}$ in liquid phase (i.e., $\mathrm{H}_{2} \mathrm{SO}_{4}$ medium) remains confusing in oxidizing carbon materials, which ranges from aggressive to moderate, and even incompetent (see Table S1 for a comparison of the literature results).

Oxidations by ferrate(VI) under solvent-free condition provide an alternative and green way for harnessing its oxidizing power. Very recently, our group discovered that the intrinsic high reactivity of $\mathrm{K}_{2} \mathrm{FeO}_{4}$ was accessible in the "dry" solid state by oxidizing small-molecule substrates [42]. On this basis, $\mathrm{K}_{2} \mathrm{FeO}_{4}$ solids were applied for the oxidation of CNTs under mechanical milling, and effective surface functionalization was achieved. However, the dry chemistry of ferrate(VI) is still poorly understood and the mechanism of $\mathrm{K}_{2} \mathrm{FeO}_{4}$ oxidation on carbon materials remains unclear. A critical question is whether $\mathrm{K}_{2} \mathrm{FeO}_{4}$ is reactive enough to open the inert $\mathrm{C}=\mathrm{C}$ bonds in carbon lattices.

Herein, we scrutinize the reactivity of ferrate(VI) in liquid phase and solid state using various forms of carbon materials as substrates, as depicted in Fig. 1. Fullerene $C_{60}$ is selected as a probe molecule, and four typical nanocarbons with diverse physical structures and different defect degrees are further used to test the reactivity. Our systematic results provide a rational understanding on the performance of this attractive oxidizer in materials chemistry.

\section{Experimental Section}

\subsection{Preparation of $\mathrm{K}_{2} \mathrm{FeO}_{4}$}

The purity of $\mathrm{K}_{2} \mathrm{FeO}_{4}$ should be examined before it is used for oxidizing carbon materials. The commercially supplied $\mathrm{K}_{2} \mathrm{FeO}_{4}$ products from several manufactures only have actual purities of $20 \%$ or below, although the declared purities are $>90 \%$. Therefore, we synthesized $\mathrm{K}_{2} \mathrm{FeO}_{4}$ according to the literature [43] and purified them by recrystallization as described in our previous work [42]. Preparation details were provided in Supporting Information (SI).

\subsection{Oxidation of Carbon Materials}

\subsubsection{Liquid-Phase $\mathrm{K}_{2} \mathrm{FeO}_{4}$ Oxidation}

Oxidation of $\mathrm{C}_{60} .30 \mathrm{mg}$ of $\mathrm{C}_{60}$ was slowly added to $20.0 \mathrm{~mL}$ of sulfuric acid (95-98\%) in a 50-mL two-necked flask under argon atmosphere, and the dispersion was sonicated for $30 \mathrm{~min}$. Then $2.5 \mathrm{~g}$ of $\mathrm{K}_{2} \mathrm{FeO}_{4}$ was slowly added to the flask under argon flow at $0{ }^{\circ} \mathrm{C}$, and the reaction mixture was stirred at $60{ }^{\circ} \mathrm{C}$ for $12 \mathrm{~h}$. The resulting dispersion was diluted in $500 \mathrm{~mL}$ of cold water and stirred for $30 \mathrm{~min}$. 

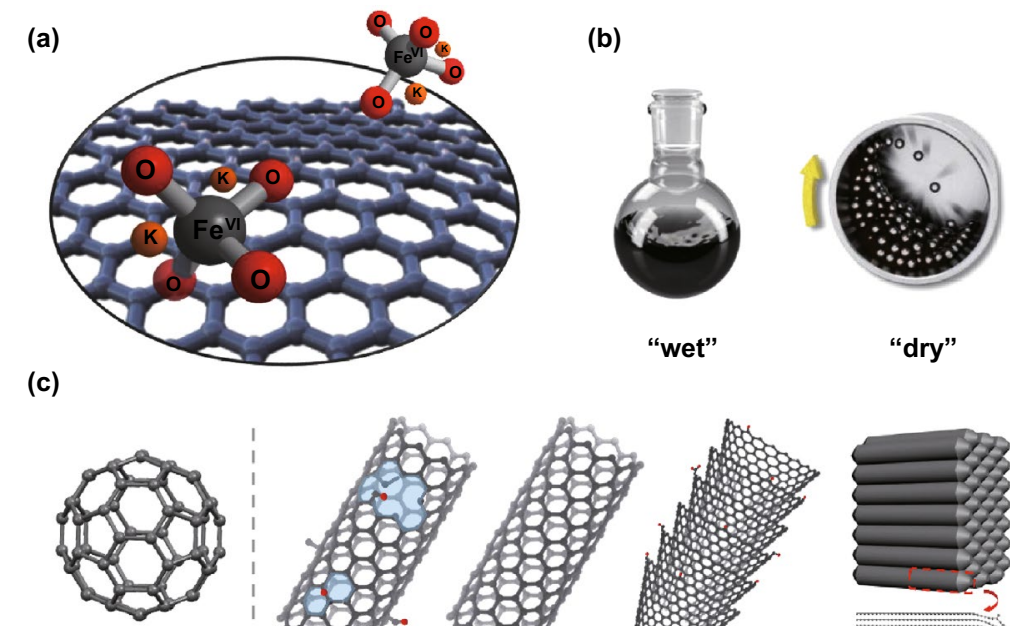

$\mathrm{C}_{60}$
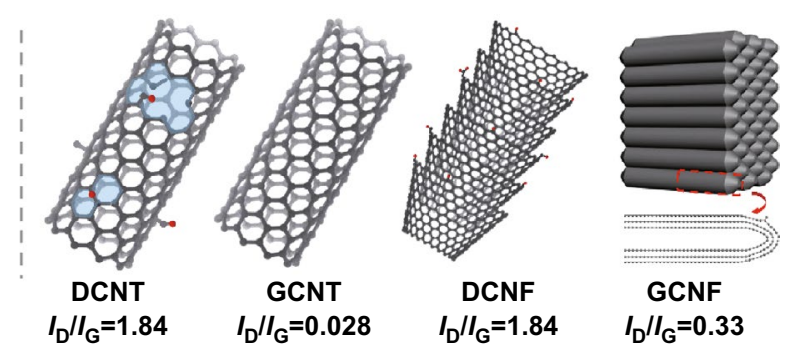

Fig. 1 a A schematic representation of $\mathrm{K}_{2} \mathrm{FeO}_{4}$-carbon reaction system. b Depiction of two reaction conditions (in $\mathrm{H}_{2} \mathrm{SO}_{4}$ medium or by solidstate ball milling). c Structural models of the carbon nanomaterials used in this study: $\mathrm{C}_{60}$, defective CNT (DCNT), graphitized CNT (GCNT), defective and graphitized carbon nanofibers (DCNF and GCNF). $I_{\mathrm{D}} / I_{\mathrm{G}}$ refers to the relative intensity of D to G band in Raman spectrum, taken as a measure of defect degree

The solid was obtained by centrifugation $(12,000 \mathrm{rpm})$, followed by washing in sequence with $2 \mathrm{M} \mathrm{HCl}$ (several times to remove $\left.\mathrm{Fe}^{3+}\right)$, ultrapure water $(18.2 \mathrm{M} \Omega \mathrm{cm})$ and ethanol. The product was finally dried at $60{ }^{\circ} \mathrm{C}$ in a vacuum oven.

Liquid-phase oxidation treatments of other carbon materials were described in SI.

\subsubsection{Solid-State $\mathrm{K}_{2} \mathrm{FeO}_{4}$ Oxidation}

Oxidation of $\mathrm{C}_{60} .100 \mathrm{mg}$ of $\mathrm{C}_{60}$ and $2.5 \mathrm{~g}$ of $\mathrm{K}_{2} \mathrm{FeO}_{4}$ were mixed together by brief grinding in an agate mortar. The mixture was then introduced into a $50-\mathrm{mL}$ stainless milling jar together with $26 \mathrm{~g}$ of 5-mm-diameter stainless steel balls (ball-to-powder weight ratio 10:1). Ball milling was performed at a rotational speed of $250 \mathrm{rpm}$ for $6 \mathrm{~h}$ or $180 \mathrm{rpm}$ for $2 \mathrm{~h}$ in a horizontal planetary ball milling (WXQM-2L, Tecan Powder). The jar was opened every 30 min to break up the mixture materials if they were agglomerated or adhered to the sidewall during milling process. The obtained solid was washed by centrifugation $(12,000 \mathrm{rpm})$ in sequence with
$2 \mathrm{M} \mathrm{HCl}$ (several times to remove $\mathrm{Fe}^{3+}$ ), ultrapure water $(18.2 \mathrm{M} \Omega \mathrm{cm})$ and ethanol. The product was finally dried at $60{ }^{\circ} \mathrm{C}$ in a vacuum oven.

Solid-state oxidation treatments of other carbon materials were described in SI.

\subsection{Characterization}

The purity of $\mathrm{K}_{2} \mathrm{FeO}_{4}$ sample was tested by spectrophotometry, X-ray diffraction (XRD) and ${ }^{57} \mathrm{Fe}$ Mössbauer spectroscopy $\left({ }^{57} \mathrm{Co}(\mathrm{Pd})\right.$ source). Chemical structure of $\mathrm{C}_{60}$ samples was determined using matrix-assisted laser desorption/ionization Fourier transform ion cyclotron resonance mass spectrum (MALDI-FTICR MS) and ${ }^{1} \mathrm{H}$ NMR (400 MHz). Oxidation degrees of carbon materials were analyzed using X-ray photoelectron spectroscopy (XPS, binding energies were calibrated with respect to $\mathrm{C} 1 \mathrm{~s}$ peak at $284.6 \mathrm{eV}$ ) and thermogravimetry (TG, $10^{\circ} \mathrm{C} \min ^{-1}, \mathrm{~N}_{2}$ ). Defect degree and morphology were characterized by Raman spectra, scanning electron microscopy and transmission electron microscopy (SEM and TEM). Details of instruments and test conditions were described in SI. 


\section{Results and Discussion}

\subsection{Purity Analysis of $\mathrm{K}_{2} \mathrm{FeO}_{4}$}

$\mathrm{K}_{2} \mathrm{FeO}_{4}$ oxidizer used in our experiments has a purity of $95 \%$, as determined by spectrophotometry. XRD pattern confirmed single-phase character of the solid (Fig. S1), and the ${ }^{57} \mathrm{Fe}$ Mössbauer spectrum proved $97.6 \%$ relative content of ferrate(VI) (Fig. S2, Table S2).

\subsection{Probing the Reactivity Using $\mathrm{C}_{60}$ Molecules}

We first probed the reactivity of $\mathrm{K}_{2} \mathrm{FeO}_{4}$ using $\mathrm{C}_{60}$. As a special kind of carbon material with defined molecular structure, $\mathrm{C}_{60}$ can provide directive and reliable assessment results. The spherical cage of $\mathrm{C}_{60}$ confers an excess of strain to $\mathrm{C}=\mathrm{C}$ bonds, inducing a unique $\mathrm{sp}^{2.28}$ hybridization of the carbon atoms with a pyramidalization angle $\theta_{\mathrm{P}}$ of $11.6^{\circ}[44$, 45]. This endows $C_{60}$ with a moderate reactivity: inerter than that of the $\mathrm{sp}^{3}-\mathrm{C}$ atoms $\left(\theta_{\mathrm{P}}=19.5^{\circ}\right)$ appearing as defects on carbon surface and more active than that of the $\mathrm{sp}^{2}$-C atoms with smaller $\theta_{\mathrm{P}} \mathrm{s}$ in graphite $\left(\theta_{\mathrm{P}}=0^{\circ}\right)$ and most other carbon materials (e.g., CNTs and CNFs) [46]. In addition, the smallmolecule property of $\mathrm{C}_{60}$ allows its product structure to be easily determined by standard organic analytical methods such as mass and NMR spectroscopy.

After the liquid-phase oxidation, $\mathrm{C}_{60}$ products showed scarcely any changes with respect to the pristine sample, even with a large excess of $\mathrm{K}_{2} \mathrm{FeO}_{4}$ (ca. 300 mol equivalent) and long reaction time (up to $12 \mathrm{~h}$ ). They displayed black color, poor water dispersibility and good solubility in toluene (Fig. $2 b$ inset). The product structure was determined by MALDI-FTICR mass spectrometry (MS). Except from the prominent peak at $\mathrm{m} / \mathrm{z} 720.000$ (intact $\mathrm{C}_{60}$ ions), no newly produced ion peaks were observed (Fig. S3), which unambiguously showed $\mathrm{K}_{2} \mathrm{FeO}_{4}$ in liquid phase was not reactive enough to oxidize $\mathrm{C}_{60}$.

In contrast, $\mathrm{K}_{2} \mathrm{FeO}_{4}$ efficiently oxidized $\mathrm{C}_{60}$ in the "dry" way. By only $1 \sim 2$-h treatment, the majority of products became hydrophilic, yielding a thick dark-brown aqueous layer after phase separation with toluene (Fig. 2b inset), which was ascribed to the generation of oxygen-containing groups. With prolonged reaction time, the purple toluene layer faded and the aqueous phase gradually became

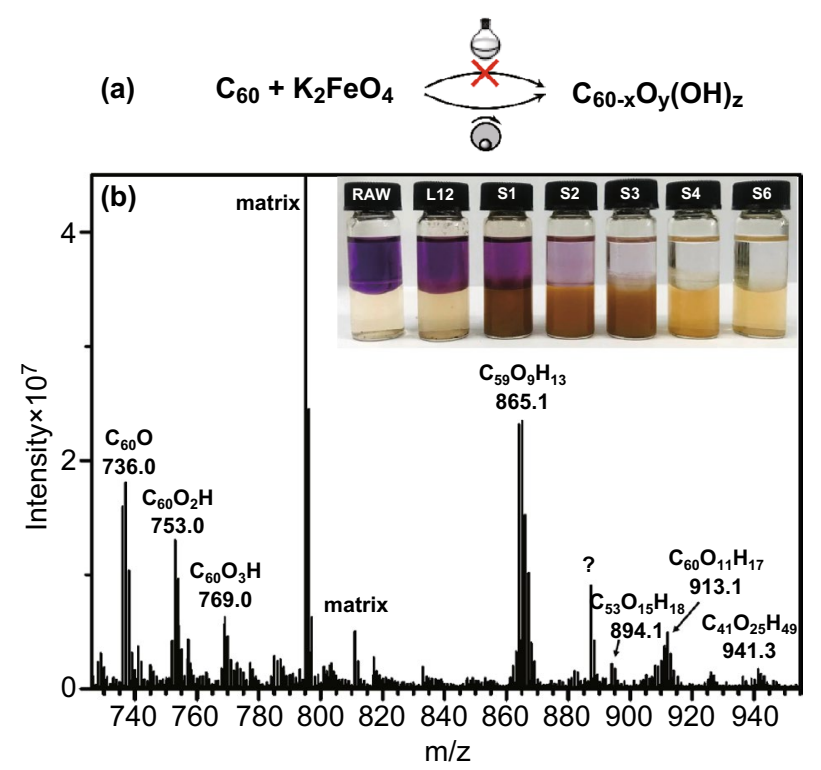

Fig. 2 a Comparison of $\mathrm{C}_{60}$ oxidation results by $\mathrm{K}_{2} \mathrm{FeO}_{4}$ in liquid phase and solid state. $\mathbf{b}$ MS of oxidized $\mathrm{C}_{60}$ products by 6-h solidstate reaction (ball milling at $250 \mathrm{rpm}$ ). Inset shows photographs of $\mathrm{C}_{60}$ samples in toluene-water biphasic system. L12 refers to the product by 12-h liquid-phase treatment, and S1-6 refers to that by solidstate reaction for $1-6 \mathrm{~h}$

yellowish transparent, indicating continuous oxidative transformation and increasing oxidation degree [47]. The product MS showed many ion clusters above $\mathrm{m} / \mathrm{z} 720.004$, clearly separated by multiples of $16(\mathrm{O})$ and $17(\mathrm{OH})$ mass units. Various oxidized species were identified, including $\mathrm{C}_{60} \mathrm{O}$ (736.000), $\mathrm{C}_{60} \mathrm{O}_{2} \mathrm{H}$ (753.003), $\mathrm{C}_{60} \mathrm{O}_{3} \mathrm{H}$ (768.998) and $\mathrm{C}_{60} \mathrm{O}_{11} \mathrm{H}_{17}$ (913.078). The attachment of $-\mathrm{OH}$ groups onto $\mathrm{C}_{60}$ cage was also confirmed by ${ }^{1} \mathrm{H}$ NMR (Fig. S4).

The oxidation treatment not only introduced oxygen and hydroxy entities to $\mathrm{C}_{60}$ cages, but also gave rise to cageopened products such as $\mathrm{C}_{59} \mathrm{O}_{9} \mathrm{H}_{13}$ (865.057), $\mathrm{C}_{53} \mathrm{O}_{15} \mathrm{H}_{18}$ (894.063) and $\mathrm{C}_{41} \mathrm{O}_{25} \mathrm{H}_{49}$ (941.248). We suspected it was the mechanical force that broke the molecular cages, but the production of these broken cages was not remarkably depressed (62\% vs. $50 \%$, relative quantifications by MS) when the energy input was substantially decreased (from $250 \mathrm{rpm} \times 6 \mathrm{~h}$ to $180 \mathrm{rpm} \times 2 \mathrm{~h}$ ). On the other hand, when $\mathrm{K}_{2} \mathrm{FeO}_{4}$ was replaced by a non-oxidizing isomorphous salt $\mathrm{K}_{2} \mathrm{SO}_{4}$, the amount of cage-opened products was dramatically reduced ( $20 \%$ by $\mathrm{K}_{2} \mathrm{SO}_{4}$ vs. $62 \%$ by $\mathrm{K}_{2} \mathrm{FeO}_{4}$, $250 \mathrm{rpm} \times 6 \mathrm{~h}$ ). These results suggested that the intrinsic reactivity of $\mathrm{K}_{2} \mathrm{FeO}_{4}$ in solid state was strong enough to 
cleave the $\mathrm{C}=\mathrm{C}$ bonds of $\mathrm{C}_{60}$ (presumably by the addition of $\mathrm{Fe}^{\mathrm{VI}}=\mathrm{O}$ with $\mathrm{C}=\mathrm{C}$ bonds).

Based on the results provided by $\mathrm{C}_{60}$ probe, it is clear that: (1) $\mathrm{K}_{2} \mathrm{FeO}_{4}$ in $\mathrm{H}_{2} \mathrm{SO}_{4}$ environment can hardly attack the $s p^{2.28}-\mathrm{C}$ of strained $\mathrm{C}=\mathrm{C}$ bonds. As a reasonable inference, it would not be able to oxidize the $\mathrm{C}=\mathrm{C}$ bonds that are inerter in most carbon materials (such as the graphite in debate); (2) $\mathrm{K}_{2} \mathrm{FeO}_{4}$ in solid state can open the strained $\mathrm{C}=\mathrm{C}$ bonds and even consume the $\mathrm{C}$ atoms in the skeleton. Therefore, the active defective sites on carbon surface could be readily oxidized by such dry chemistry, as has been observed in our previous work on DCNT functionalization [42].

\subsection{Further Testing the Reactivity Using Nanocarbons}

It is still uncertain whether (1) the liquid-phase $\mathrm{K}_{2} \mathrm{FeO}_{4}$ takes effect in the oxidation of active defects and (2) solid-state $\mathrm{K}_{2} \mathrm{FeO}_{4}$ is oxidizing enough for less strained $\mathrm{C}=\mathrm{C}$ bonds. To address these issues, the reactivity of $\mathrm{K}_{2} \mathrm{FeO}_{4}$ was further determined using nanocarbons including CNTs and CNFs, in both defective and graphitized types (Figs. 1c, S5 and S12). Specifically, DCNT contains rich numbers of surface defects (adatoms, vacancies, cracks, etc.) [48], exhibiting an $I_{\mathrm{D}} / I_{\mathrm{G}}$ ratio of up to 1.84 , while GCNT has a well-graphitized, nearly defectless surface [49-51] with a very low $I_{\mathrm{D}} / I_{\mathrm{G}}$ of 0.028 . DCNF is made of stacked graphene "cups" exposing large amounts of edge sites on the outer shells $[52,53]$ giving a high $I_{\mathrm{D}} / I_{\mathrm{G}}$ of 1.84 , while GCNF, constructed by close packing of carbon rods, features edge-closed loops formed by high-temperature graphitization [54] with a much lower $I_{\mathrm{D}} / I_{\mathrm{G}}$ of 0.33 .

\subsection{1 $\mathrm{K}_{2} \mathrm{FeO}_{4}$ in Liquid Phase Could Only Oxidize Surface Defects}

The oxidizing ability of $\mathrm{K}_{2} \mathrm{FeO}_{4}$ in liquid phase was found to be modest: the oxidation was efficient to the defective nanocarbons while inoperative to the graphitized ones. To be specific, DCNTs treated by $\mathrm{K}_{2} \mathrm{FeO}_{4} / \mathrm{H}_{2} \mathrm{SO}_{4}$ for $2 \mathrm{~h}$ showed good aqueous dispersibility, in contrast to the insoluble raw material (Fig. 3a). The surface O/C ratio (detected by XPS) showed an increase from 3.5\% (raw) to $9.1 \%$ (2 h) (Fig. 3b). In addition, TG weight loss (Fig. 3c), originated from thermolysis of functional groups on surfaces, also supported the increased oxygen content on 2-h-treated DCNTs. However,
GCNTs after treatment (up to $8 \mathrm{~h}$ ) showed properties that were substantially unchanged compared to the raw material, including poor water dispersibility, few contents of surface oxygen and low levels of TG weight loss (Fig. 3a-c). The results of CNFs treated by $\mathrm{K}_{2} \mathrm{FeO}_{4}$ followed a similar trend with those of CNTs (Fig. 3a, e, f). Note that 8-h-treated GCNFs displayed distinguishable oxidation effects, since the so-called graphitized CNFs actually contained a certain number of defects in view of the $I_{\mathrm{D}} / I_{\mathrm{G}}$ of 0.33 .

The above results suggested that liquid-phase oxidation by $\mathrm{K}_{2} \mathrm{FeO}_{4}$ could only occur at the original defects on carbon materials. Raman spectra further confirmed that additional defects (which would arise from the reactions on $\mathrm{C}=\mathrm{C}$ bonds) were not produced during the reaction process, as reflected by the almost unchanged $I_{\mathrm{D}} / I_{\mathrm{G}}$ values for both unoxidized nanocarbons and the oxidatively modified ones (Fig. 3d, g). As a result, carbon nanomaterials were protected from structural damage during the oxidation treatment, as shown by SEM and TEM images in Figs. 4 and S5. These results supported the nondestructive oxidation of CNTs reported by Zhang and Xu [39].

According to the reactivity clarified above, $\mathrm{K}_{2} \mathrm{FeO}_{4}$ in $\mathrm{H}_{2} \mathrm{SO}_{4}$ medium can only provide a slight oxidizing effect on graphite, which arises from reactions at the edge sites of graphene sheets, and the basal planes are unlikely to be affected. This explains why $\mathrm{K}_{2} \mathrm{FeO}_{4} / \mathrm{H}_{2} \mathrm{SO}_{4}$ method is unsuitable for preparing graphene oxide as pointed out by Sofer et al. [36]. On the other hand, when bulk graphite sample was replaced by nanographite platelets with high ratio of edge-to-plane sites (Fig. S6), distinct oxidizing effects were observed: the surface $\mathrm{O} / \mathrm{C}$ ratio was increased to $6.8 \%$ with discernible - $\mathrm{COOH}$ peak in $\mathrm{C}$ 1s spectrum after 8-h treatment (Fig. S7), which manifested again the ability of $\mathrm{K}_{2} \mathrm{FeO}_{4}$ to oxidize the defective sites on carbon surface.

\subsection{2 $\mathrm{K}_{2} \mathrm{FeO}_{4}$ in Solid State Could Open the Inert $\mathrm{C}=\mathrm{C}$ Bonds}

$\mathrm{K}_{2} \mathrm{FeO}_{4}$ in solid state was able to oxidize not only the defective nanocarbons but also the graphitized ones. For nanocarbons which got negligible oxidation in liquid phase, effective oxidative modifications were achieved in the solid state; and in cases when liquid-phase oxidation was successful, the solid-state reactions would provide higher levels of oxidation (Fig. 3, Table S4). Such strong oxidizing effect can be 
(a)
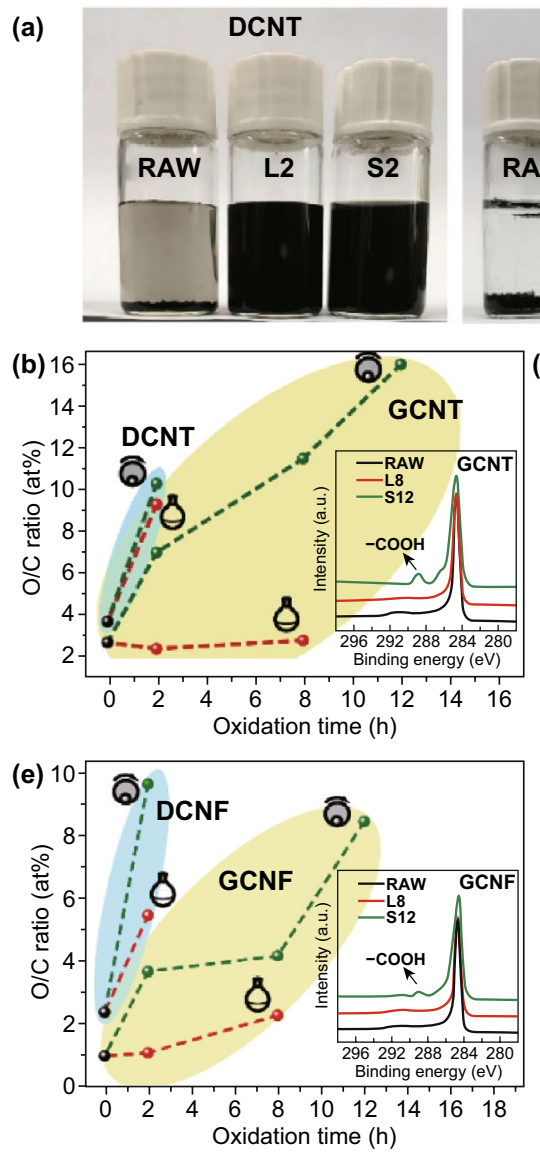
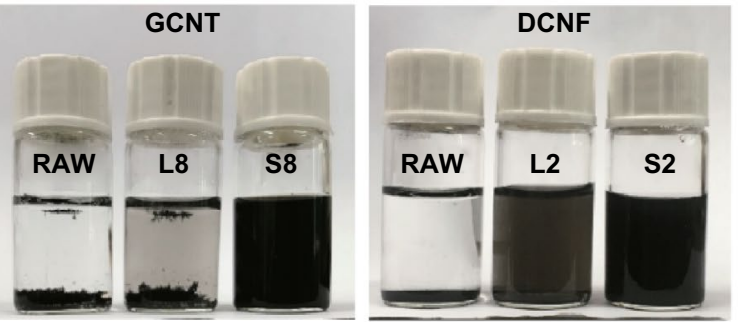

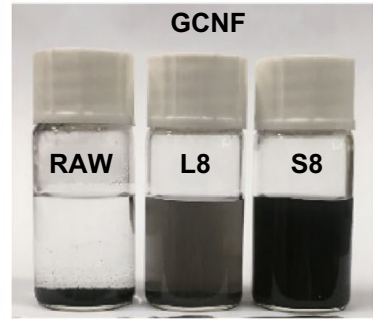

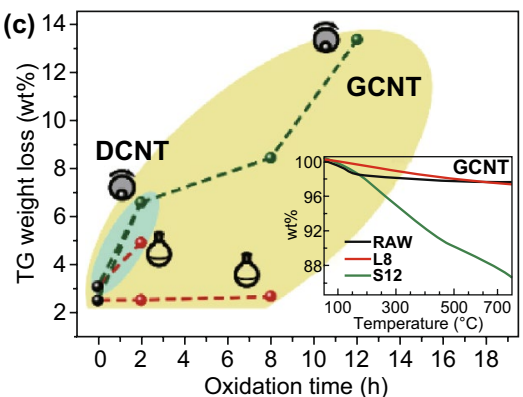
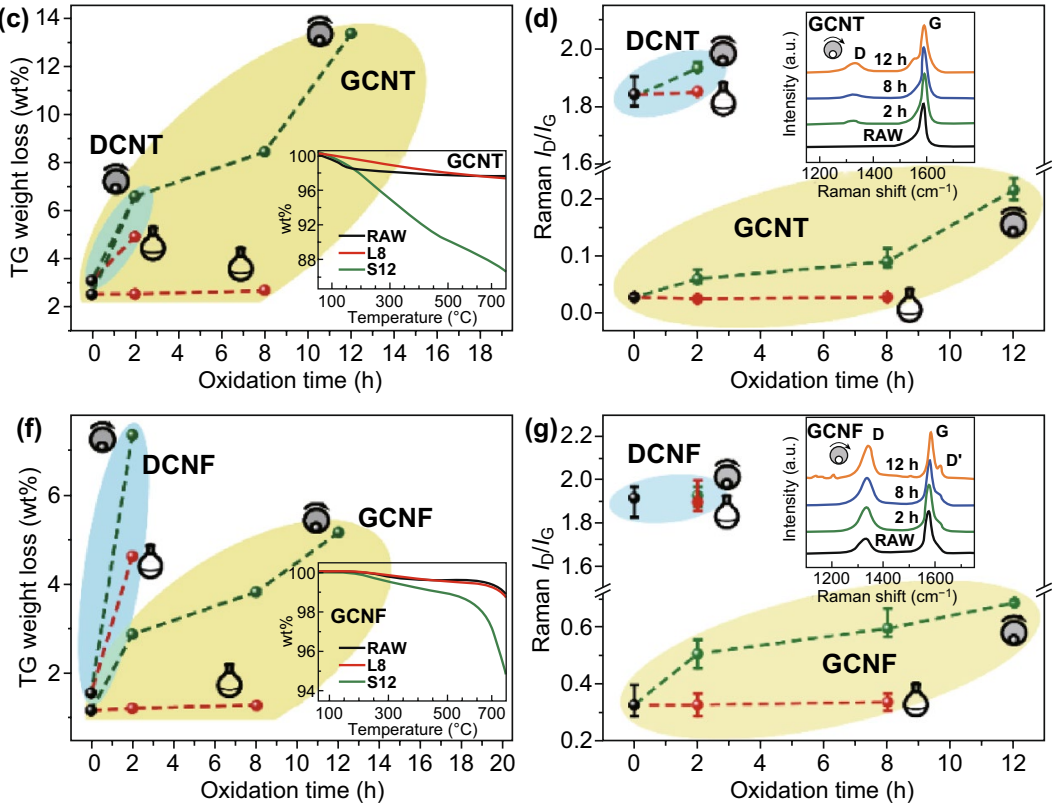

(h)

DCNT

GCNT

DCNF

GCNF

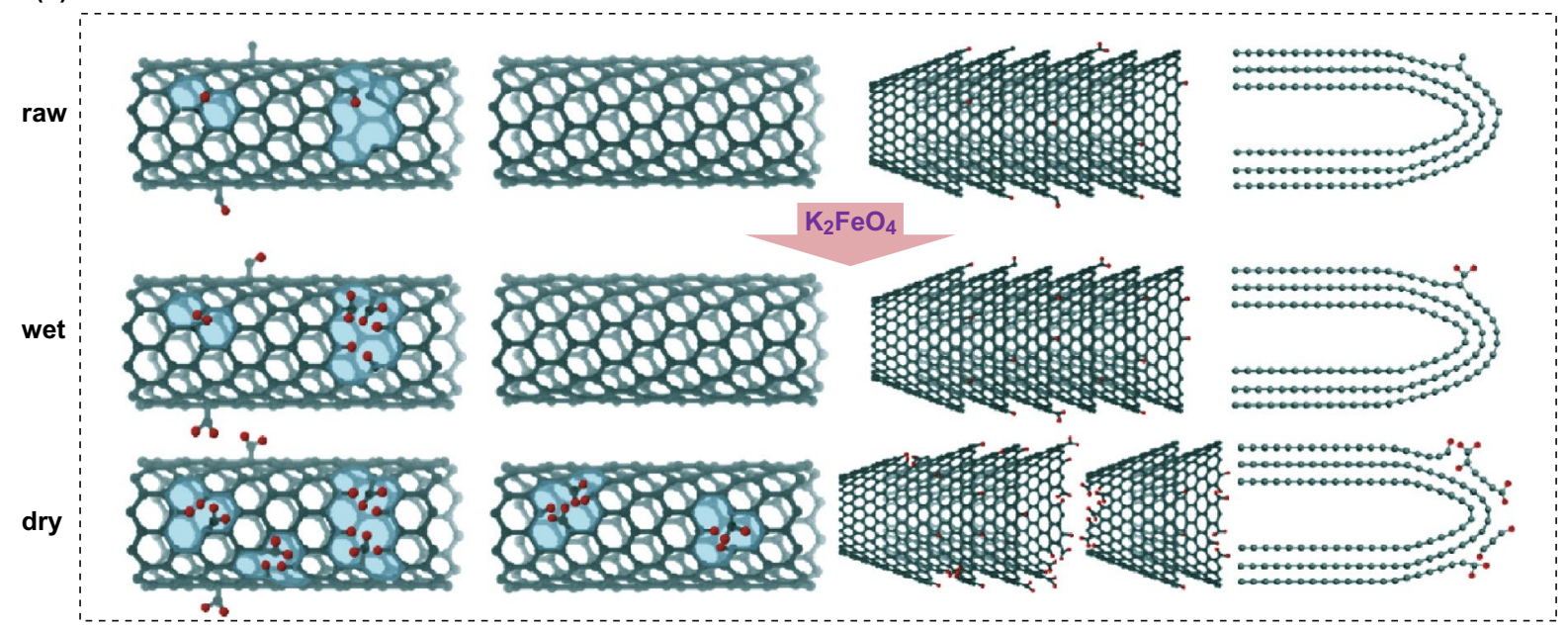

Fig. 3 Oxidation results of various nanocarbons by $\mathrm{K}_{2} \mathrm{FeO}_{4}$ in liquid phase and solid state. If applicable, water-dispersible portions of the products were isolated for measurements to better show the oxidation effects. a Photographs of $0.1 \mathrm{mg} \mathrm{mL}^{-1}$ samples in water that had been sonicated for $5 \mathrm{~min}$ and settled for 3 days. b, e XPS O/C ratios (inset: representative C 1s spectra). c, f TG weight losses (inset: representative TG curves). d, $\mathbf{g} I_{\mathrm{D}} / I_{\mathrm{G}}$ ratios (inset: representative Raman spectra). $\mathbf{h}$ A depiction of the effects of $\mathrm{K}_{2} \mathrm{FeO}_{4}$ oxidation on nanocarbons in liquid phase and solid state (oxygen atoms are shown in red). (Color figure online) 

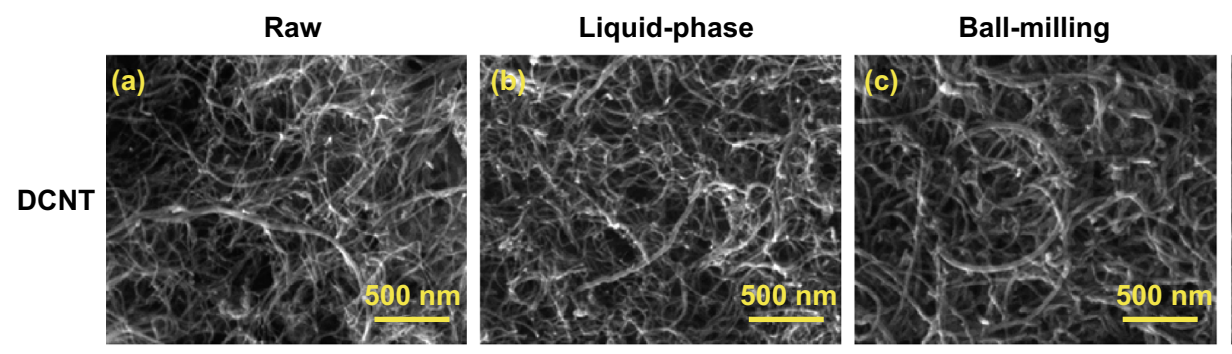

\section{Stronger ball-milling}
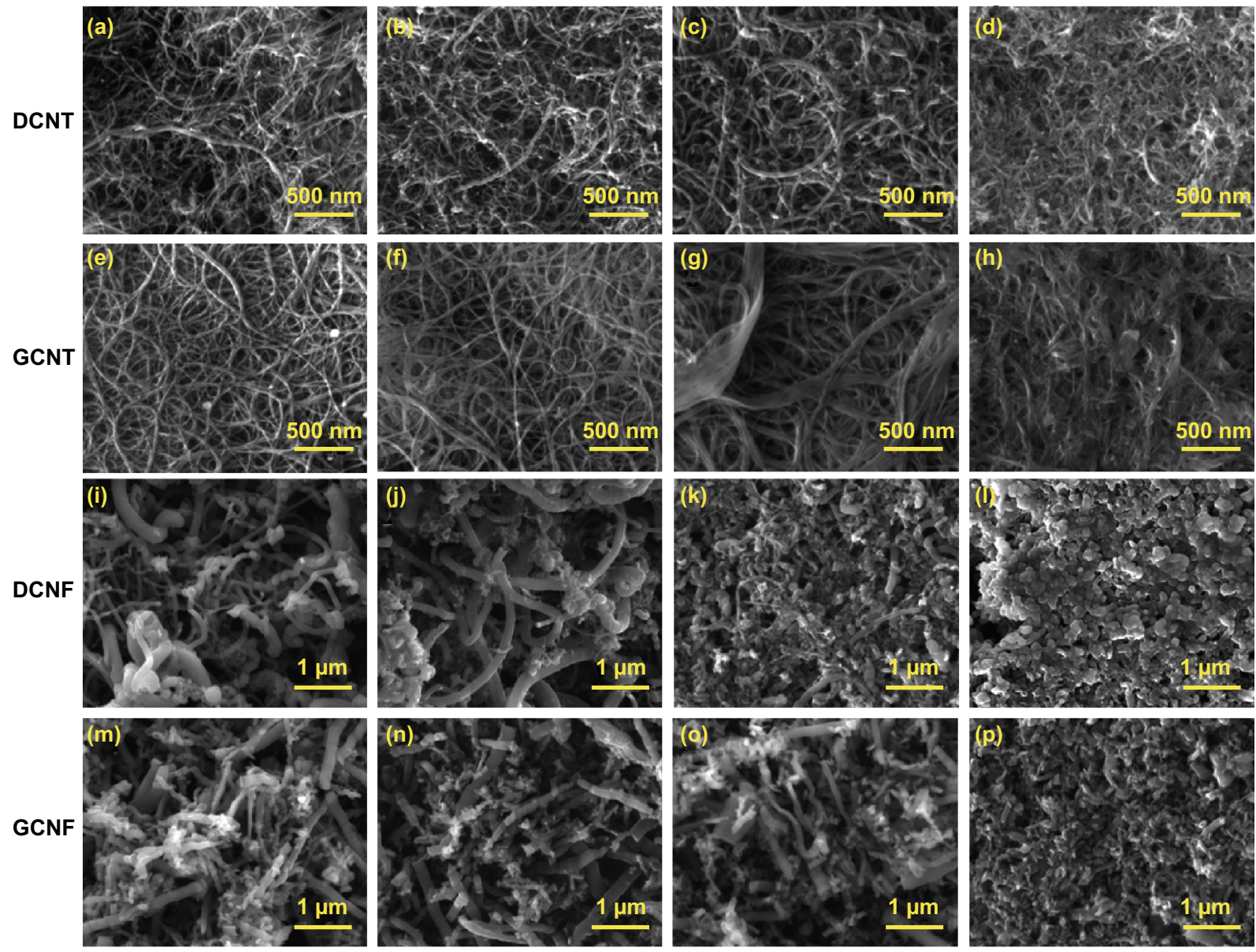

Fig. 4 SEM images of carbon nanomaterials under different reaction conditions. a-d DCNTs: a raw, b L2, c S2 at 250 rpm, d S2 at 300 rpm; e-h GCNTs: e raw, f L8, $\mathbf{g}$ S8 at $250 \mathrm{rpm}, \mathbf{h}$ S8 at $300 \mathrm{rpm}$; i-l DCNFs: $\mathbf{i}$ raw, j L2, $\mathbf{k}$ S2 at $100 \mathrm{rpm}, \mathbf{1}$ S2 at $250 \mathrm{rpm}$; m-p GCNFs: m raw, $\mathbf{n}$ L8, o S2 at $100 \mathrm{rpm}, \mathbf{p ~ S 1 2}$ at $100 \mathrm{rpm}$

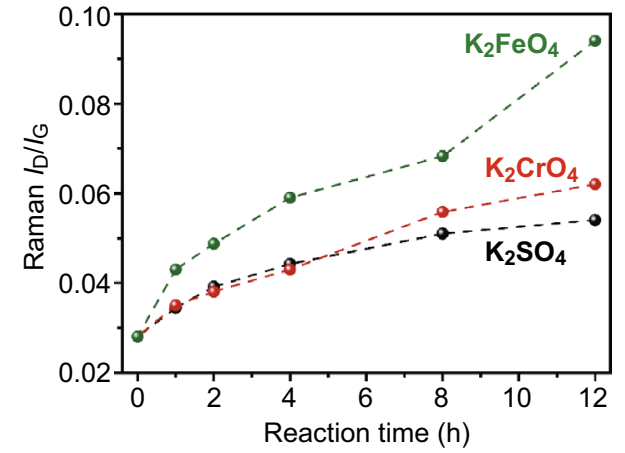

Fig. $5 I_{\mathrm{D}} / I_{G}$ ratio results of GCNTs by solid-state milling $(250 \mathrm{rpm})$ with three isomorphous salts. A comparison of Raman spectra is shown in Fig. S13 interpreted as the ability to open the inert $\mathrm{C}=\mathrm{C}$ bonds in carbon lattice, as indicated by the evidently increased $I_{\mathrm{D}} / I_{\mathrm{G}}$ values after solid-state oxidations (Fig. 3d, g).

To figure out whether the bond breakage is merely a result of physical effect by ball milling or contributed from the chemical reactivity of $\mathrm{K}_{2} \mathrm{FeO}_{4}$ solids, two isomorphous salts, non-oxidizing $\mathrm{K}_{2} \mathrm{SO}_{4}$ and weakly oxidizing $\mathrm{K}_{2} \mathrm{CrO}_{4}$, were also used in the dry reaction system for comparison. We used a mild ball milling condition of $250 \mathrm{rpm}$ as suggested by the $\mathrm{C}_{60}$ experiments, and the nearly defectless GCNTs were taken as substrate. As displayed in Fig. 5, GCNTs during ball milling with $\mathrm{K}_{2} \mathrm{SO}_{4}$ showed gentle increase in the defect content with time $\left(I_{\mathrm{D}} / I_{\mathrm{G}}\right.$ only increased 0.025 after $12 \mathrm{~h}$ ), which was reasonably induced by mechanical force. $\mathrm{K}_{2} \mathrm{CrO}_{4}$ treatment had a comparable effect with slightly 
more defects at long reaction times. In contrast, $\mathrm{K}_{2} \mathrm{FeO}_{4}$ led to efficient introduction of defects, producing much higher defect degrees than the other two salts at the same condition. Therefore, $\mathrm{K}_{2} \mathrm{FeO}_{4}$ solids possessed sufficient oxidizing ability to directly open the inert $\mathrm{C}=\mathrm{C}$ bonds under the mechanochemical conditions. It was no surprise that $\mathrm{C}_{60}$ cages were easily damaged by such dry oxidation.

\subsection{Discussion}

Our systematic studies using elaborately selected carbon materials clearly reveal two distinct oxidizing activities of ferrate(VI) depending on the reaction environment. Their different oxidation effects on four nanocarbons are depicted in Fig. 3h.

Liquid-phase oxidation only works for the defective sites, which means the reactivity of ferrate(VI) is largely depressed in $\mathrm{H}_{2} \mathrm{SO}_{4}$ medium and becomes much weaker than that of the commonly used oxidizers (e.g., $\mathrm{KMnO}_{4}$ and $\mathrm{HNO}_{3}$ ) for carbon materials. Such modest reactivity limits its scope of application, but may be desirable for functionalizing defective carbon materials, where effective surface oxidation can be achieved with no risk of disturbing the carbon structure or morphology.

In solid state, ferrate(VI) readily oxidizes the surface defects and its oxidizing power is strong enough to break the inert $\mathrm{C}=\mathrm{C}$ bonds in carbon lattice. Solid-state ferrate(VI) oxidation is thus generally applicable for introducing oxygenated groups (e.g., $-\mathrm{COOH}$ ) onto various carbon surface. In addition, the mechanical force involved in reaction can favor the oxidation performance by producing more defects. On the other hand, the mechanochemical conditions (e.g., rotation speed, ball type and time) must be optimized to avoid undesired structural damage, especially for fragile materials like CNFs (see Figs. 4 and S8-S11 for detailed studies on the effect of reaction condition on product structure).

\section{Conclusions}

By using molecular and nanoforms of carbon as substrates, we unraveled the reactivity of ferrate(VI) in oxidizing carbon materials. The theoretically strong oxidizing power of ferrate(VI) is largely depressed in $\mathrm{H}_{2} \mathrm{SO}_{4}$ medium, yielding a modest reactivity that only oxidizes the active defects on carbon surface. This liquid-phase ferrate(VI) oxidation can be used as a gentle approach to functionalizing defect-rich carbon materials with the advantage of protecting structural integrity. Ferrate(VI) in solid state releases a high oxidizing power that is capable of opening the inert $\mathrm{C}=\mathrm{C}$ bonds in carbon lattice, making it generally applicable to introduce oxygenated groups to various carbon materials. This intrinsic strong reactivity underlies the dry chemistry of ferrate(VI) and implies its wide scope of applications in green and powerful oxidative functionalization/ transformation.

These two distinct oxidizing abilities could also apply to other kinds of materials. Considering the emerging role of high-valent iron compounds (represented by ferrate(VI)) in materials science, understanding their reactivity in different conditions is of fundamental importance for guiding their applications.

Acknowledgements The authors acknowledge the financial support from National Key Research and Development Program of China (2017YFA0207500), National Natural Science Foundation of China $(51673114,51973111)$ and Shanghai Science and Technology Committee (17ZR1447300). We thank the Instrumental Analysis Center of Shanghai Jiao Tong University. We are grateful to Prof. Hang Wang for providing the MALDI-FTICR MS tests. We thank Prof. Dong-Mei Jiang (East China Normal University) for providing the ${ }^{57} \mathrm{Fe}$ Mössbauer spectroscopy test.

Open Access This article is licensed under a Creative Commons Attribution 4.0 International License, which permits use, sharing, adaptation, distribution and reproduction in any medium or format, as long as you give appropriate credit to the original author(s) and the source, provide a link to the Creative Commons licence, and indicate if changes were made. The images or other third party material in this article are included in the article's Creative Commons licence, unless indicated otherwise in a credit line to the material. If material is not included in the article's Creative Commons licence and your intended use is not permitted by statutory regulation or exceeds the permitted use, you will need to obtain permission directly from the copyright holder. To view a copy of this licence, visit http://creativecommons.org/licenses/by/4.0/.

Electronic supplementary material The online version of this article (https://doi.org/10.1007/s40820-019-0353-2) contains supplementary material, which is available to authorized users.

\section{References}

1. J.F. Berry, E. Bill, E. Bothe, S.D. George, B. Mienert, F. Neese, K. Wieghardt, An octahedral coordination complex of 
iron(VI). Science 312(5782), 1937-1941 (2006). https://doi. org/10.1126/science. 1128506

2. J.J. Scepaniak, C.S. Vogel, M.M. Khusniyarov, F.W. Heinemann, K. Meyer, J.M. Smith, Synthesis, structure, and reactivity of an iron(V) nitride. Science 331(6020), 1049-1052 (2011). https://doi.org/10.1126/science.1198315

3. J. Hohenberger, K. Ray, K. Meyer, The biology and chemistry of high-valent iron-oxo and iron-nitrido complexes. Nat. Commun. 3, 720 (2012). https://doi.org/10.1038/ncomms1718

4. V.K. Sharma, Ferrate(VI) and ferrate(V) oxidation of organic compounds: kinetics and mechanism. Coord. Chem. Rev. 257(2), 495-510 (2013). https://doi.org/10.1016/j. ccr.2012.04.014

5. J. Torres-Alacan, U. Das, A.C. Filippou, P. Vohringer, Observing the formation and the reactivity of an octahedral iron(V) nitrido complex in real time. Angew. Chem. Int. Ed. 52(49), 12833-12837 (2013). https://doi.org/10.1002/ anie. 201306621

6. M. Ghosh, K.K. Singh, C. Panda, A. Weitz, M.P. Hendrich, T.J. Collins, B.B. Dhar, S. Sen Gupta, Formation of a room temperature stable $\mathrm{Fe}(\mathrm{V})(\mathrm{O})$ complex: reactivity toward unactivated C-H bonds. J. Am. Chem. Soc. 136(27), 95249527 (2014). https://doi.org/10.1021/ja412537m

7. P.J. Chirik, An $\mathrm{Fe}^{\mathrm{VI}}$ nitride: there is plenty of room at the top! Angew. Chem. Int. Ed. 45(42), 6956-6959 (2006). https ://doi.org/10.1002/anie.200603056

8. H. Schmidbaur, The history and the current revival of the oxo chemistry of iron in its highest oxidation states: $\mathrm{Fe}^{\mathrm{VI}}$ $\mathrm{Fe}^{\mathrm{VIII}}$. Z. Anorg. Allg. Chem. 644(12-13), 536-559 (2018). https://doi.org/10.1002/zaac.201800036

9. D. Ghernaout, M.W. Naceur, Ferrate(VI): in situ generation and water treatment - a review. Desalin. Water Treat. 30(13), 319-332 (2012). https://doi.org/10.5004/dwt.2011.2217

10. V.K. Sharma, Oxidation of inorganic contaminants by ferrates (VI, V, and IV) - kinetics and mechanisms: a review. J. Environ. Manag. 92(4), 1051-1073 (2011). https://doi. org/10.1016/j.jenvman.2010.11.026

11. J.-Q. Jiang, B. Lloyd, Progress in the development and use of ferrate(VI) salt as an oxidant and coagulant for water and wastewater treatment. Water Res. 36(6), 1397-1408 (2002). https://doi.org/10.1016/S0043-1354(01)00358-X

12. R.H. Wood, The heat, free energy and entropy of the ferrate(VI) ion. J. Am. Chem. Soc. 80(9), 2038-2041 (1958). https://doi.org/10.1021/ja01542a002

13. V.K. Sharma, R. Zboril, R.S. Varma, Ferrates: greener oxidants with multimodal action in water treatment technologies. Acc. Chem. Res. 48(2), 182-191 (2015). https://doi. org/10.1021/ar5004219

14. J.-Q. Jiang, Advances in the development and application of ferrate(VI) for water and wastewater treatment. J. Chem. Technol. Biotechnol. 89(2), 165-177 (2014). https://doi. org/10.1002/jctb.4214

15. V.K. Sharma, Potassium ferrate(VI): an environmentally friendly oxidant. Adv. Environ. Res. 6(2), 143-156 (2002). https://doi.org/10.1016/s1093-0191(01)00119-8
16. M. Feng, C. Jinadatha, T.J. McDonald, V.K. Sharma, Accelerated oxidation of organic contaminants by ferrate(VI): the overlooked role of reducing additives. Environ. Sci. Technol. 52(19), 11319-11327 (2018). https://doi.org/10.1021/acs. est. 8 b03770

17. M. Feng, J.C. Baum, N. Nesnas, Y. Lee, C.-H. Huang, V.K. Sharma, Oxidation of sulfonamide antibiotics of sixmembered heterocyclic moiety by Ferrate(VI): kinetics and mechanistic insight into $\mathrm{SO}_{2}$ extrusion. Environ. Sci. Technol. 53(5), 2695-2704 (2019). https://doi.org/10.1021/acs. est.8b06535

18. V.K. Sharma, L. Chen, R. Zboril, Review on high valent $\mathrm{Fe}^{\mathrm{VI}}$ (Ferrate): a sustainable green oxidant in organic chemistry and transformation of pharmaceuticals. ACS Sustain. Chem. Eng. 4(1), 18-34 (2016). https://doi.org/10.1021/acssuschem eng.5b01202

19. L. Delaude, P. Laszlo, A novel oxidizing reagent based on potassium ferrate(VI). J. Org. Chem. 61(18), 6360-6370 (1996). https://doi.org/10.1021/jo960633p

20. P.K. Tandon, S.B. Singh, M. Srivastava, Synthesis of some aromatic aldehydes and acids by sodium ferrate in presence of copper nano-particles adsorbed on K 10 montmorillonite using microwave irradiation. Appl. Organomet. Chem. 21(4), 264-267 (2007). https://doi.org/10.1002/aoc.1198

21. J. Zhang, J. Li, Y. Tang, L. Lin, M. Long, F. Yang, Selective conversion of biomass-derived precursor 5-hydroxymethylfurfural to 2,5-furandicarboxylic acid by ferrate (VI) oxidation. J. Biobased Mater. Bio. 9(5), 502-508 (2015). https:// doi.org/10.1166/jbmb.2015.1547

22. S. Licht, Energetic iron(VI) chemistry: the super-iron battery. Science 285(5430), 1039-1042 (1999). https://doi. org/10.1126/science.285.5430.1039

23. S. Licht, A high capacity Li-ion cathode: the Fe(III/VI) superiron cathode. Energies 3(5), 960-972 (2010). https://doi. org/10.3390/en3050960

24. M.V. Simičić, M.I. Čekerevac, L.N. Nikolić-Bujanović, I.Z. Veljković, M.Z. Zdravković, M.M. Tomić, Influence of nonstoichiometric binary titanium oxides addition on the electrochemical properties of the barium ferrate plastic-bonded cathode for super-iron battery. Electrochim. Acta 247, 516-523 (2017). https://doi.org/10.1016/j.electacta.2017.07.056

25. R. Sarma, A.M. Angeles-Boza, D.W. Brinkley, J.P. Roth, Studies of the di-iron(VI) intermediate in ferrate-dependent oxygen evolution from water. J. Am. Chem. Soc. 134(37), 15371-15386 (2012). https://doi.org/10.1021/ja304786s

26. L. Ma, W.W. Lam, P.K. Lo, K.C. Lau, T.C. Lau, $\mathrm{Ca}^{2+}$-induced oxygen generation by $\mathrm{FeO}_{4}{ }^{2-}$ at $\mathrm{pH}$ 9-10. Angew. Chem. Int. Ed. 55(9), 3012-3016 (2016). https://doi.org/10.1002/ anie. 201510156

27. G. Chen, W.W.Y. Lam, P.K. Lo, W.L. Man, L. Chen, K.C. Lau, T.C. Lau, Mechanism of water oxidation by ferrate(VI) at $\mathrm{pH}$ 7-9. Chem. Eur. J. 24(70), 18735-18742 (2018). https://doi. org/10.1002/chem.201803757

28. J.S. Greer, Oxygen generating candles. U.S. Patent 5049306 (Sep. 17, 1991) 
29. B.F. Monzyk, Apparatus and methods of providing diatomic oxygen $\left(\mathrm{O}_{2}\right)$ using ferrate(VI)-containing compositions. U.S. Patent 8944048 B2 (Feb. 3, 2015)

30. M.B. Sassin, A.N. Mansour, K.A. Pettigrew, D.R. Rolison, J.W. Long, Electroless deposition of conformal nanoscale iron oxide on carbon nanoarchitectures for electrochemical charge storage. ACS Nano 4(8), 4505-4514 (2010). https:// doi.org/10.1021/nn100572a

31. H. Wang, J. Wang, Q. Zou, W. Liu, C. Wang, W. Huang, Surface treatment using potassium ferrate for separation of polycarbonate and polystyrene waste plastics by froth flotation. Appl. Surf. Sci. 448, 219-229 (2018). https://doi. org/10.1016/j.apsusc.2018.04.091

32. Y. Gong, D. Li, C. Luo, Q. Fu, C. Pan, Highly porous graphitic biomass carbon as advanced electrode materials for supercapacitors. Green Chem. 19(17), 4132-4140 (2017). https://doi. org/10.1039/c7gc01681f

33. M.M. Najafpour, S.M. Hosseini, Toward a nanosized iron based water-oxidizing catalyst. Int. J. Hydrogen Energy 41(48), 22635-22642 (2016). https://doi.org/10.1016/j.ijhyd ene.2016.08.106

34. X. Duan, D. Wang, G. Qian, J.C. Walmsley, A. Holmen, D. Chen, X. Zhou, Fabrication of K-promoted iron/carbon nanotubes composite catalysts for the Fischer-Tropsch synthesis of lower olefins. J. Energy Chem. 25(2), 311-317 (2016). https:// doi.org/10.1016/j.jechem.2016.01.003

35. L. Peng, Z. Xu, Z. Liu, Y. Wei, H. Sun, Z. Li, X. Zhao, C. Gao, An iron-based green approach to 1 -h production of singlelayer graphene oxide. Nat. Commun. 6, 5716 (2015). https:// doi.org/10.1038/ncomms6716

36. Z. Sofer, J. Luxa, O. Jankovsky, D. Sedmidubsky, T. Bystron, M. Pumera, Synthesis of graphene oxide by oxidation of graphite with ferrate(VI) compounds: myth or reality? Angew. Chem. Int. Ed. 55(39), 11965-11969 (2016). https://doi. org/10.1002/anie.201603496

37. S. Mura, Y. Jiang, I. Vassalini, A. Gianoncelli, I. Alessandri et al., Graphene oxide/iron oxide nanocomposites for water remediation. ACS Appl. Nano Mater. 1(12), 6724-6732 (2018). https://doi.org/10.1021/acsanm.8b01540

38. A. Romero, M.P. Lavin-Lopez, L. Sanchez-Silva, J.L. Valverde, A. Paton-Carrero, Comparative study of different scalable routes to synthesize graphene oxide and reduced graphene oxide. Mater. Chem. Phys. 203, 284-292 (2018). https://doi. org/10.1016/j.matchemphys.2017.10.013

39. Z.Y. Zhang, X.C. Xu, Nondestructive covalent functionalization of carbon nanotubes by selective oxidation of the original defects with $\mathrm{K}_{2} \mathrm{FeO}_{4}$. Appl. Surf. Sci. 346, 520-527 (2015). https://doi.org/10.1016/j.apsusc.2015.04.026

40. H. Yu, B. Zhang, C. Bulin, R. Li, R. Xing, High-efficient synthesis of graphene oxide based on improved hummers method. Sci. Rep. 6, 36143 (2016). https://doi.org/10.1038/srep36143

41. H. Yu, D. Ye, T. Butburee, L. Wang, M. Dargusch, Green synthesis of porous three-dimensional nitrogen-doped graphene foam for electrochemical applications. ACS Appl. Mater.
Interfaces 8(4), 2505-2510 (2016). https://doi.org/10.1021/ acsami.5b09030

42. Z.Y. Zhang, D. Ji, W. Mao, Y. Cui, Q. Wang et al., Dry chemistry of ferrate(VI): a solvent-free mchanochemical way for versatile green oxidation. Angew. Chem. Int. Ed. 57, 10949 (2018). https://doi.org/10.1002/anie.201805998

43. C. Li, X.Z. Li, N. Graham, A study of the preparation and reactivity of potassium ferrate. Chemosphere 61(4), 537-543 (2005). https://doi.org/10.1016/j.chemosphere.2005.02.027

44. R.C. Haddon, L.E. Brus, K. Raghavachari, Electronic structure and bonding in icosahedral $\mathrm{C}_{60}$. Chem. Phys. Lett. 125(5), 459-464 (1986). https://doi.org/10.1016/0009-2614(86)87079 $-8$

45. S. Niyogi, M.A. Hamon, H. Hu, B. Zhao, P. Bhowmik, R. Sen, M.E. Itkis, R.C. Haddon, Chemistry of single-walled carbon nanotubes. Acc. Chem. Res. 35(12), 1105-1113 (2002). https ://doi.org/10.1021/ar010155r

46. S.A. Hodge, M.K. Bayazit, K.S. Coleman, M.S. Shaffer, Unweaving the rainbow: a review of the relationship between single-walled carbon nanotube molecular structures and their chemical reactivity. Chem. Soc. Rev. 41(12), 4409-4429 (2012). https://doi.org/10.1039/c2cs15334c

47. K. Kokubo, K. Matsubayashi, H. Tategaki, H. Takada, T. Oshima, Facile synthesis of highly water-soluble fullerenes more than half-covered by hydroxyl groups. ACS Nano 2(2), 327-333 (2008). https://doi.org/10.1021/nn700151z

48. J.C. Charlier, Defects in carbon nanotubes. Acc. Chem. Res. 35(12), 1063-1069 (2002). https://doi.org/10.1021/ar010166k

49. Z. Yongsheng, Z. Yingchun, X. Bingshe, Z. Xueji, A.A.-G. Khalid, M. Shahid, Cobalt sulfide confined in N-doped porous branched carbon nanotubes for lithium-ion batteries. NanoMicro Lett. 11, 29 (2019). https://doi.org/10.1007/s4082 0-019-0259-Z

50. T. Zhang, Z.S. Yuan, L.H. Tan, Exact geometric relationships, symmetry breaking and structural stability for single-walled carbon nanotubes. Nano-Micro Lett. 3(4), 228-235 (2011). https://doi.org/10.3786/nml.v3i4.p228-235

51. C. Gao, J. Han, Functionalization of carbon nanotubes and other nanocarbons by azide chemistry. Nano-Micro Lett. 2(3), 213-226 (2010). https://doi.org/10.1007/bf03353643

52. J. Huang, Y. Liu, T. You, Carbon nanofiber based electrochemical biosensors: a review. Anal. Methods 2(3), 202 (2010). https://doi.org/10.1039/b9ay00312f

53. Z. Tang, Y. Zhao, Q. Lai, J. Zhong, Y. Liang, Stepwise fabrication of Co-embedded porous multichannel carbon nanofibers for high-efficiency oxygen reduction. Nano-Micro Lett. 11, 33 (2019). https://doi.org/10.1007/s40820-019-0264-2

54. S.-H. Yoon, S. Lim, S.-H. Hong, I. Mochida, B. An, K. Yokogawa, Carbon nano-rod as a structural unit of carbon nanofibers. Carbon 42(15), 3087-3095 (2004). https://doi. org/10.1016/j.carbon.2004.07.022 